\title{
sensors
}

ISSN 1424-8220

(C) 2002 by MDPI

http://www.mdpi.net/sensors

\section{Gamma Radiation Dosimetry Using Tellurium Dioxide Thin Film Structures}

\author{
Khalil Arshak* and Olga Korostynska \\ Electronic \& Computer Engineering Dept., University of Limerick, Limerick, Ireland. \\ * Author to whom correspondence should be addressed. E-mail: Khalil.Arshak@ul.ie
}

Received:3 July 2002 / Accepted:19 July 2002 / Published: 23 August 2002

\begin{abstract}
Thin films of Tellurium dioxide (TeO2) were investigated for $\gamma$-radiation dosimetry purposes. Samples were fabricated using thin film vapour deposition technique. Thin films of $\mathrm{TeO} 2$ were exposed to a $60 \mathrm{Co} \gamma$-radiation source at a dose rate of $6 \mathrm{~Gy} / \mathrm{min}$ at room temperature. Absorption spectra for $\mathrm{TeO} 2$ films were recorded and the values of the optical band gap and energies of the localized states for as-deposited and $\gamma$-irradiated samples were calculated. It was found that the optical band gap values were decreased as the radiation dose was increased. Samples with electrical contacts having a planar structure showed a linear increase in current values with the increase in radiation dose up to a certain dose level. The observed changes in both the optical and the electrical properties suggest that $\mathrm{TeO} 2$ thin film may be considered as an effective material for room temperature real time $\gamma$-radiation dosimetry.
\end{abstract}

Key words: Gamma Radiation, Dosimetry, Thin Films, Tellurium Dioxide, Optical Band Gap

\section{Introduction}

The ability to detect and perform energy-dispersive spectroscopy of high-energy radiation such as $\mathrm{X}$-rays, $\gamma$-rays, and other uncharged and charged particles has improved dramatically in recent years [20]. This is of great importance in a wide range of applications including medical imaging, industrial process monitoring, national security and treaty verification, environmental safety and remediation, and basic science. The influence of $\gamma$-radiation onto different types of thin films has been discussed earlier $[18,21]$. 
The aim of this experimental work is to investigate the changes in both the electrical and optical properties of $\mathrm{TeO}_{2}$ thin film structures under the influence of $\gamma$-radiation. Crystal structure and optical properties of $\mathrm{TeO}_{2}$ were the subjects of numerous theoretical and experimental studies [4, 12]. Tellurium dioxide belongs to the category of compounds in which all the atoms are the so-called pelements, having non-bonding valence electron pairs [4]. High-energy radiations, such as $\gamma$-rays, change the physical properties of the materials they penetrate. The changes are strongly dependent on the internal structure of the absorbed substances. It is believed that ionising radiation causes structural defects (called colour centres or oxygen vacancies in oxides) leading to their density change on the exposure to $\gamma$-rays [25]. The lowest threshold energy for displacement of in-plane atoms is known to correspond to the displacement of in-plane oxygen that is about $58 \mathrm{keV}$ [22]. The influence of radiation depends on both the dose and the parameters of the films including their thickness: the degradation is more severe for the higher dose and the thinner films [3].

\section{Experimental Procedure}

An Edwards E306A vacuum thermal coating system was used for thin films deposition. This system contains an Edwards FTM5 quartz crystal to monitor the rate of film deposition and to measure the film thickness. The quartz crystal was positioned directly above the evaporation source. The mass deposited on the quartz crystal during the evaporation alters its natural frequency of vibration. This frequency change was recorded on the meter of the film thickness monitor connected to the quartz crystal. Thus the monitor could record both the thickness and the rate of deposition corresponding to a particular frequency shift. Planar configurations having structure shown in Figure 1 were fabricated. Two layers of Aluminium having thickness of $100 \mathrm{~nm}$ and separated by a distance of $2 \mathrm{~mm}$ were deposited on suitably prepared glass substrate to act as contacts. A tungsten filament in the form of a coil with few spirals was used to hang the wires for the evaporation of Aluminium. On top of these contacts an active layer of $\mathrm{TeO}_{2}$ having the thickness of $335 \mathrm{~nm}$ was deposited from a tungsten boat at a deposition rate of $3-5 \mathrm{~nm} / \mathrm{s}$.

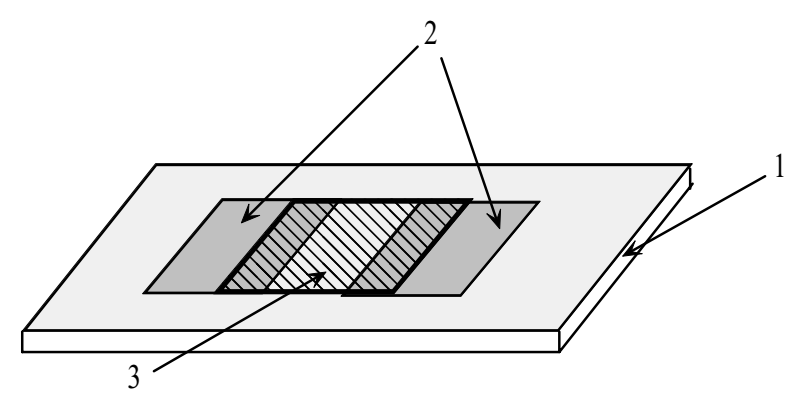

Figure 1. Planar structure used for the electrical parameters measurements, where 1 is a glass substrate, 2 - Al layers used as electrodes, 3 - layer of $\mathrm{TeO}_{2}$

Films having thickness of $50 \mathrm{~nm}$ were chosen for the absorption spectra studies, as the thicker layers of $\mathrm{TeO}_{2}$ were specularly reflective. In contrast to that thicker films were more suitable for the investigation of the electrical properties, as they exhibited greater conductivity. 
A ${ }^{60} \mathrm{Co}$ radiation source with a dose rate of $6 \mathrm{~Gy} / \mathrm{min}$ was used for exposing the samples to $\gamma$ radiation at room temperature. A set of irradiations were performed changing the exposure time and hence the dose. Current-voltage characteristics for as-deposited and $\gamma$-irradiated specimens were recorded after each exposure dose.

The absorption spectra for all the samples were recorded using CARY 1E UV-Visible Spectrophotometer.

Values of radiation damage were estimated from changes in both the electrical and optical parameters.

\section{Results And Discussion}

\section{Optical Properties}

The optical properties of a material are important, as they provide information on the electronic band structures, localized states and types of optical transitions. Thin dielectric films generally are amorphous in nature. However, it is clear from both the experimental and theoretical determinations that the atomic arrangement in terms of nearest neighbours in most amorphous solids departs only slightly from the ideal crystalline state [17]. Furthermore, since amorphous solids contain relatively rigid chemical bonds in terms, of both, the direction and the length, deviation from the crystalline state is limited. As a result of this, excessive energy would be required to bend or distort this rigidity. Therefore it is possible to assume a certain kind of short range or local order exists because of this almost rigid bond model [14]. The most important consequence of this "short-range order" is that the fundamental band structure of crystalline materials remains a valid concept for amorphous materials. The band structure and the existence of an energy gap are believed to be dependent upon the arrangement of nearest atomic neighbours and the existence of local or short-range order. Mott was the first who proposed the concept of short-range order in amorphous solids [15]. The lack of crystalline long-range order in amorphous/glassy materials is associated with a tailing of the density of states into the normally forbidden energy band [1]. Urbach and Martienssen reported the exponential dependence of the photon energy near the absorption edge [11,23]. The exponential absorption tails (referred as Urbach's energy, $\Delta E$ ) depend on temperature induced disorder, static disorder, strong ionic bonds and also on average phonon energies.

Figure 2 shows typical plots of the absorption spectra for as-deposited and $\gamma$-irradiated $\mathrm{TeO}_{2}$ films having thickness of $50 \mathrm{~nm}$. These spectra have similar shape to those recorded for powdered samples of $\mathrm{TeO}_{2}$ reported by [16]. From the picture above it is evident that the optical absorption spectral distribution is sensitive to the radiation influence. The absorption coefficient $\alpha(v)$ of the optical absorption near the band edge shows an exponential dependence on photon energy $h v$ and obeys the Urbach's empirical relation (1):

$$
\alpha(v)=\alpha_{0} \exp \left(\frac{h v}{\Delta E}\right)
$$

where $\alpha_{0}$ is a constant and $\Delta E$ is the width of the band tails of localized states. The values of $\Delta E$ were calculated from the slopes of the straight lines of $\ln \alpha$ versus $h v$ plots. Figure 3 shows the increase in 
the normalized energy of the localized states $\left(\Delta \mathrm{E}-\Delta \mathrm{E}_{0}\right) / \Delta \mathrm{E}_{0}$ with the increase in radiation dose.

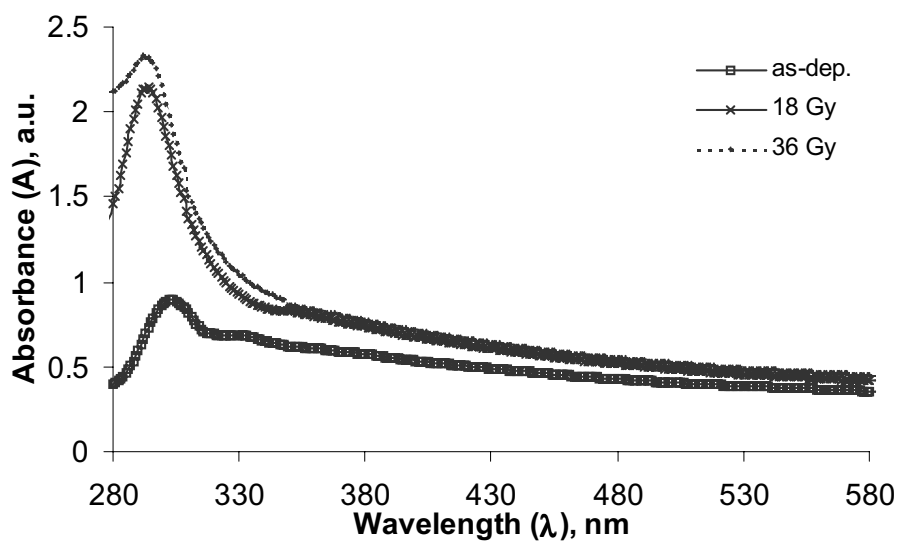

Figure 2. The absorption spectra for as-deposited and irradiated $50 \mathrm{~nm} \mathrm{TeO}_{2}$ thin films.

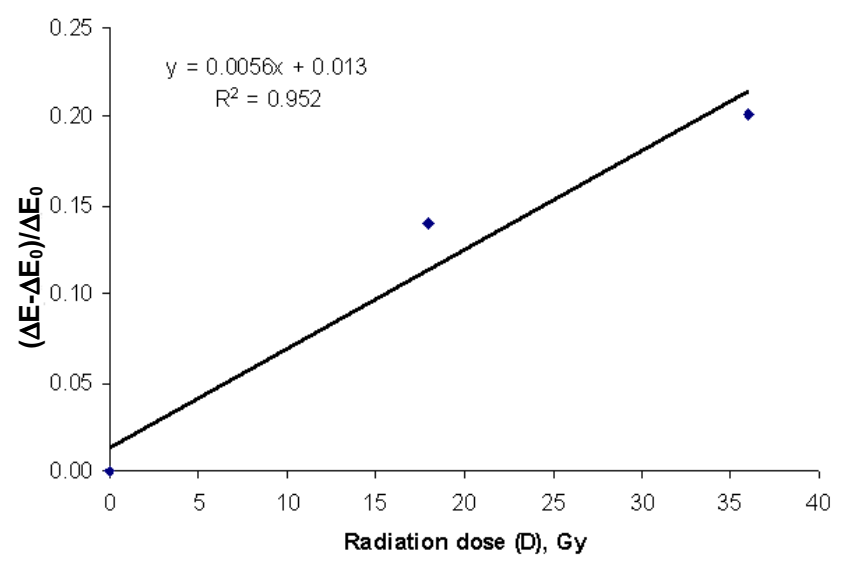

Figure 3. Increase in the normalized energy of the localized states $\left(\Delta \mathrm{E}-\Delta \mathrm{E}_{0}\right) / \Delta \mathrm{E}_{0}$ with the increase in radiation dose.

The values of the optical band gap for as-deposited and $\gamma$-irradiated films were estimated using the Mott and Davis' model [13] for the direct allowed transition using Eq.2:

$$
\alpha(v) h v=B\left(h v-E_{\text {opt }}\right)^{1 / 2}
$$

where $\alpha$ is the absorption coefficient, $E_{\text {opt }}$ is the optical energy band gap, $h v$ is the energy of the incident photons and $B$ is a constant. In this study, the calculated optical bang gap value for asdeposited films of $\mathrm{TeO}_{2}$ was found to be $3.75 \mathrm{eV}$ in contrast to the value reported by [19]. This discrepancy may be attributed to the different film fabrication techniques used. It has been reported that the optical band gap values $\mathrm{E}_{\mathrm{opt}}$ for many compound semiconductor materials change under the influence of radiation exposure [2, 7, 9]. Arshak et al [2] have studied the influence of $\gamma$-radiation onto the optical properties of screen printed $\mathrm{NiO}$ thick films. It has been reported that optical band gap for these films have decreased from $2.05 \mathrm{eV}$ to $1.65 \mathrm{eV}$ with the increase in radiation dose up to the level of $240 \mathrm{~Gy}$. Figure 4 demonstrates a decrease in the value of the optical energy gap $\mathrm{E}_{\mathrm{opt}}$ for $\mathrm{TeO}_{2}$ thin films as a result of $\gamma$-radiation influence. 


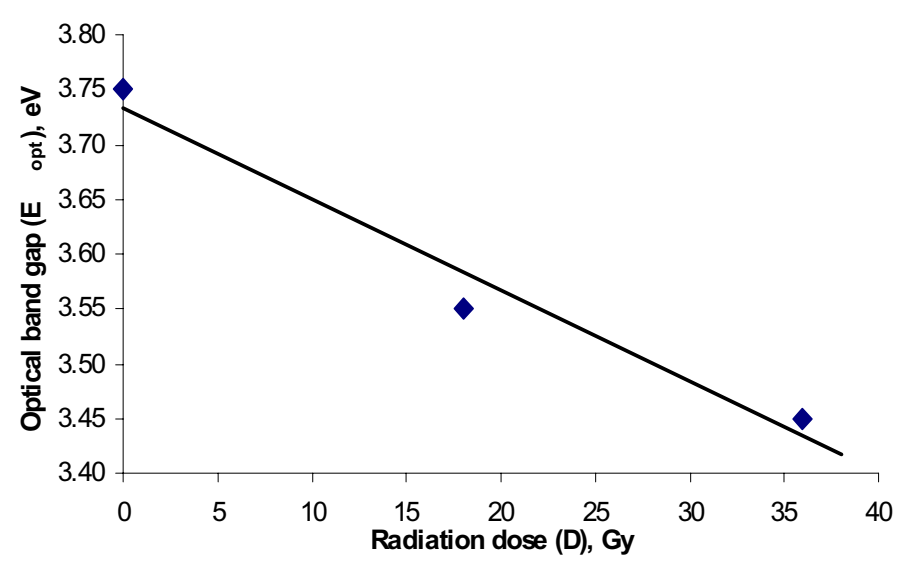

Figure 4. Decrease in the optical band gap $\left(E_{o p t}\right)$ values as a result of $\gamma$-radiation influence.

The variation of the optical energy gap with electron irradiation energies and doses can be explained as the change in the degree of disorder. From the density-of-state model, it is known that $\mathrm{E}_{\text {opt }}$ decreases with an increasing degree of disorder of the amorphous phase [10]. At this stage one may expect that a band tail be, probably, created due to irradiation. The decrease in $\mathrm{E}_{\text {opt }}$ leads to a shift in the band tail $\Delta \mathrm{E}$ towards the higher energy region and hence the values of $\Delta \mathrm{E}$ calculated by Eq.1 are expected to increase as the radiation dose is increased. Our experimental results are in good agreement with theoretical considerations.

\section{Electrical Properties}

Figure 5 shows the plots of current-voltage characteristics that were recorded for as-deposited and $\gamma$-irradiated $\mathrm{TeO}_{2}$ thin film samples with contacts having planar structure. The exposed dose was increased in steps of $18 \mathrm{~Gy}$. The value of the current was observed to increase with the increase in the radiation dose up to a level of 72 Gy. Figure 6 shows dependence of the normalized current $\left(\mathrm{I}-\mathrm{I}_{0}\right) / \mathrm{I}_{0}$ versus radiation dose under an applied voltage of $2 \mathrm{~V}$ for $\mathrm{TeO}_{2}$ thin films.

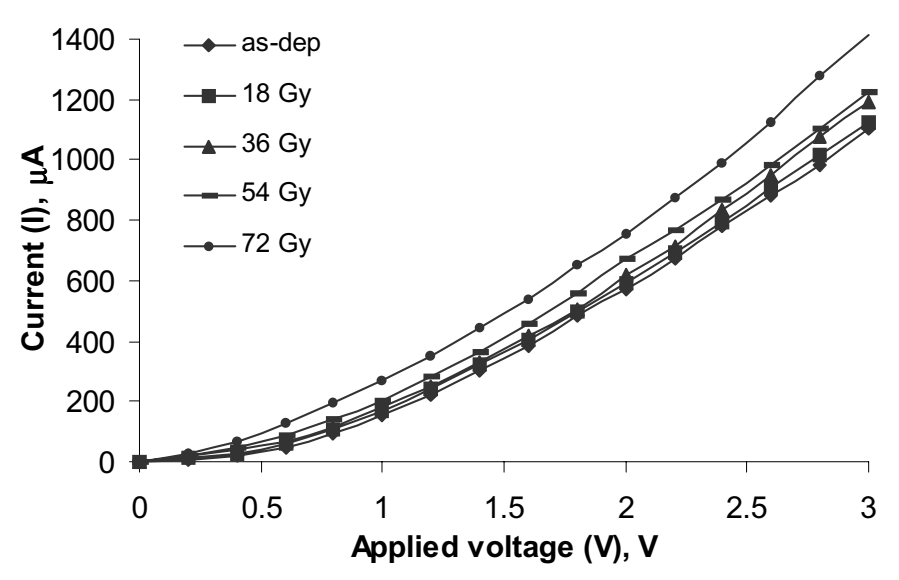

Figure 5. Plots of current-voltage characteristics that were recorded for as-deposited and $\gamma$ irradiated $\mathrm{TeO}_{2}$ samples with a planar type structure. 


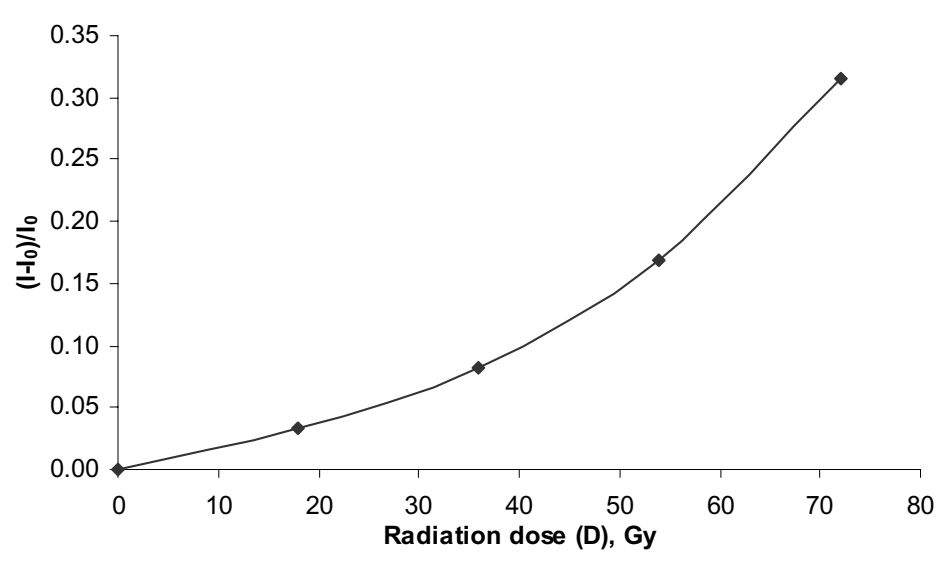

Figure 6. Dependence of the normalized current $\left(\mathrm{I}-\mathrm{I}_{0}\right) / \mathrm{I}_{0}$ versus radiation dose under an applied voltage of $2 \mathrm{~V}$ for $\mathrm{TeO}_{2}$ thin films.

While irradiation usually leads to the creation of structural defects, the healing effect of irradiation is also known [5, 24]. Metals made of powders preliminary irradiated by electrons or gammairradiation are characterized by the absence of big pores and fine homogeneous grain structure. The average sizes of grains being 4-5 times lower than that in conventional technology [24].

Ionising radiation has been found to be widely applicable in modifying the structure and properties of polymers, and can be used to tailor the performance of either bulk materials or surfaces [5]. Such a healing occurs due to radiation-stimulated recombination of intrinsic (pre-existing) defects or to radiation-stimulated ordering of initially disordered phase [22]. This is known as the small-dose effect, because it occurs at low doses when the concentration of the induced defects does not exceed the concentration of intrinsic defects. However, the healing mechanism is consistent with foregoing observations, as recombination of intrinsic defects reduces the resistivity.

Meanwhile, the actual number of defects may be much smaller than the number of displacements due to spontaneous and thermal recombination (re-ordering). It would be interesting in this connection to perform irradiation and supplementary measurements of transport properties at low temperatures to prevent re-ordering.

The behaviour of both the electrical and the optical properties of the studied specimens under the influence of $\gamma$-radiation was found to be similar to the dose response of most materials used in thermoluminescence dosimetry [8]. They usually show a linear, then supralinear, followed by saturating response and further increase in dose leads to their damage.

The small sensitivity in either the electrical or the optical properties of material to radiation or fluence $(\Phi)$ in the saturation region has been attributed to the generation of high structural disorder [6]. This damage may create a large enough concentration of localized levels, which reduces the sensitivity of parameters to further irradiation. High concentration of dangling bonds makes disordered semiconductors insensitive to doping or irradiation $[6,14]$.

Under the rudimentary model of the charge transfer, the Fermi level in the irradiated structure should shift upwards with respect to its position in the non-irradiated structure, filling the holes states in plane bands [25]. For $\mathrm{TeO}_{2}$ thin films shifting the Fermi energy level under the influence of radiation has resulted in decrease in optical band gap $\mathrm{E}_{\text {opt }}$ value (Figure 4). In turn, filling the holes 
states could give an explanation to the observed increase in values of the normalized current $\left(\mathrm{I}-\mathrm{I}_{0}\right) / \mathrm{I}_{0}$ with the increase in radiation dose (Figure 6). This approach clarifies the observed correlation between changes in both the electrical and the optical properties caused by radiation.

\section{Conclusion}

The possibility of using Tellurium dioxide thin films as a sensitive material for $\gamma$-radiation was explored. Films were fabricated using the thermal vacuum deposition technique. Samples were exposed to a ${ }^{60} \mathrm{Co} \gamma$-radiation source at a dose rate of $6 \mathrm{~Gy} / \mathrm{min}$. The characteristics were recorded after a fixed exposure time. Absorption spectra for $50 \mathrm{~nm} \mathrm{TeO}$ thin films were recorded and the values of the optical band gap and energy of the localized states were calculated. It was found that the optical properties were highly affected by the exposure to $\gamma$-radiation, e.g. optical band gap values showed a decrease and values of energy of the localized states showed an increase as radiation dose was increased. Samples having planar type configuration were used to trace the radiation-induced changes in the electrical properties of $\mathrm{TeO}_{2}$ thin films. An increase in the values of normalized current with the increase in radiation dose was observed up to a dose level of $72 \mathrm{~Gy}$.

The linear response to radiation in both the electrical and optical properties of $\mathrm{TeO}_{2}$ thin film was observed up to a certain dose level. These structures are therefore considered to be an effective material for room temperature real time $\gamma$-radiation dosimetry.

\section{Acknowledgments}

This work was performed with the assistance of AMT Ireland in the University of Limerick, as part of the RADSENAD project (ref. PRP00/AMT/06), which is sponsored by the Enterprise Ireland Development Funds.

\section{References}

1. Abay, B.; Guder, H.S.; Yogurtchu, Y.K. Urbach-Martienssen's tails in layered semiconductor GaSe. Solid State Communications 1999, 112, 489-494.

2. Arshak, K.; Korostynska, O.; Harris, J. $\gamma$-radiation dosimetry using screen printed Nickel oxide thick films. MIEL' 2002 Conference Proceedings, 2002.

3. Tanassova, E.; Paskaleva, A.; Konakova, R.; Spassov, D.; Mitin, V. F. Influence of $\gamma$-radiation on thin $\mathrm{Ta}_{2} \mathrm{O}_{5}$-Si structures. Microelectronics Journal 2001, 32, 553-562.

4. Champarnaud-Mesjard, J.C.; Blanchandin, S.; Thomas, P.; Mirgorodsky, A.; Merle-Méjean, T.; Frit, B. Crystal structure. Raman spectrum and lattice dynamics of a new metastable form of tellurium dioxide: $\gamma-\mathrm{TeO}_{2}$. Journal of Physics and Chemistry of Solids 2000, 61, 1499-1507.

5. Clough, R.L. High-energy radiation and polymers: A review of commercial processes and emerging applications. Nuclear Instruments and Methods in Physics Res. Sec. B 2001, 185, 8-33. 
6. Croitoru, N.; Gubbini, E.; Rancoita, P.G.; Rattaggi, M.; Seidman, A. Influence of damage caused by $\mathrm{Kr}$ ions and neutrons on electrical properties of silicon detectors. Nuclear Instruments and Methods in Physics, Res. Sec. A 1999, 426, 477-485.

7. El-Muraikhi, M. Effect of electric field on optical properties of post gamma-irradiated lithium potassium sulphate crystals. Materials Letters 2001, 51, 19-26.

8. Horowitz, Y.S. Theory of thermo-luminescence gamma dose response: The unified interaction model. Nuclear Instruments and Methods in Physics Research Section B 2001, 184, 68-84.

9. Ibrahim, A.M.; Soliman, L.I. Effect of $\gamma$-irradiation on optical and electrical properties of $\mathrm{Se}_{1-}$ ${ }_{\mathrm{x}} \mathrm{Te}_{\mathrm{x}}$. Radiation Physics and Chemistry 1998, 53, 469-475.

10. Kurik, M.V. Urbach rule. Physica Status Solidi A 1971, 8, 9-45.

11. Martienssen, H.W. Journal of Physics and Chemistry of Solids 1979, 2, 257.

12. Mirgorodsky, A.P.; Merle-Méjean, T.; Champarnaud, J.-C.; Thomas, P.; Frit, B. Dynamics and structure of $\mathrm{TeO}_{2}$ polymorphs: model treatment of paratellurite and tellurite; Raman scattering evidence for new $\gamma$ - and $\delta$-phases. Journal of Physics and Chemistry of Solids 2000, 61, 501-509.

13. Mott, N. F.; Davis, E. A. Electronic Process in Non-crystalline Materials, $2^{\text {nd }}$ ed, Oxford, UK: Clarendon Press, 1979.

14. Mott, N. Conduction in Non-Crystalline Materials, Oxford Science Publications, Clarendon Press, Oxford 1993, 83-102.

15. Mott, N.F. Phil. Mag. 1969, 19, 835.

16. Park, J.-H.; Woodward, P.M. Synthesis, structure and optical properties of two new Perovskites: $\mathrm{Ba}_{2} \mathrm{Bi}_{2 / 3} \mathrm{TeO}_{6}$ and $\mathrm{Ba}_{3} \mathrm{Bi}_{2} \mathrm{TeO}_{9}$. International Journal of Inorganic Materials 2000, 2, 153-166.

17. Perrem, R.L. Thin film strain gauge. An investigation into the properties of the mixed oxide $\mathrm{V}_{2} \mathrm{O}_{5^{-}}$ $\mathrm{Bi}_{2} \mathrm{O}_{3}, \mathrm{PhD}$ thesis, University of Limerick, 1993.

18. Phillips, G.W.; Readshaw, A.K.; Brown, G.O.; Weiss, R.G.; Guardala, N.A.; Price, J.L.; Mueller, S.C.; Moscovitch, M. Observation of radiation effects on three-dimensional optical random-accessmemory materials for use in radiation dosimetry. Applied Radiation and Isotopes 1999, 50, 875881.

19. Prakash, G.V.; Rao, D.N.; Bhatnagar, A.K. Linear optical properties of niobium-based tellurite glasses. Solid State Communications 2001, 119, 39-44.

20. Schlesinger, T.E.; Toney, J.E.; Yoon, H.; Lee, E.Y.; Brunett, B. A.; Franks, L.; James, R.B. Cadmium zinc telluride and its use as a nuclear radiation detector material. Materials Science and Engineering, R: Reports 2001, 32, 103-189.

21. Shpotyuk, O.I. Amorphous chalcogenide semicon-ductors for dosimetry of high-energy ionising radiation. Radiation Physics and Chemistry 1995, 46, 1279-1282.

22. Tolpygo, S.K.; Lin, J.-Y.; Gurvitch, M.; Hou, S.Y.; Phillips, J.M. T ${ }_{c}$ enhancement by low energy electron irradiation and the influence of chain disorder on resistivity and Hall coefficient in $\mathrm{YBa}_{2} \mathrm{Cu}_{3} \mathrm{O}_{7}$ thin films. Physica C: Superconductivity 1996, 269, 207-219.

23. Urbach, F. Phys. Rev. 1953, 92, 1324.

24. Zaykin, Yu. A.; Aliyev, B.A. Radiation effects in high-disperse metal media and their application in powder metallurgy. Radiation Physics and Chemistry 2002, 63, 227-230. 
25. Zhu, R.Y. Radiation damage in scintillating crystals. Nuclear Instruments and Methods in Physics Research Section A: Accelerators, Spectrometers, Detectors and Associated Equipment 1998, 413, 297-311.

Sample Availability: Available from the authors.

(C) 2002 by MDPI (http://www.mdpi.net). Reproduction is permitted for noncommercial purposes. 